\title{
Sterile searches with Liquid Argon at FNAL
}

\author{
Gian Luca Raselli* \\ on behalf of the ICARUS Collaboration \\ INFN Sezione di Pavia \\ E-mail: gianluca.raselli@pv.infn.it
}

\begin{abstract}
A new Short Baseline Neutrino (SBN) experiment is in preparation at FNAL to definitely prove or exclude the existence of non-standard neutrino oscillations into sterile states. The program foresees the deployment along the Booster Neutrino Beam (BNB) of three detectors, all based on the liquid-argon time projection chamber technique. This technology has been taken to full maturity with ICARUS T600, which will act as far detector after the completion of the overhauling at CERN and its transportation to FNAL. The program foresees the data taking for three years.
\end{abstract}

Neutrino Oscillation Workshop

4 - 11 September, 2016

Otranto (Lecce, Italy)

\footnotetext{
* Speaker.
} 


\section{The Short Baseline Neutrino (SBN) program at FNAL}

Experimental observations of neutrino oscillations have so far established a consistent paradigm consisting of three neutrino flavors $\left(v_{e}, v_{\mu}, v_{\tau}\right)$ resulting from the mixing of three mass eigenstates $\left(v_{1}, v_{2}, v_{3}\right)$. Nonetheless, some experiments have reported short-baseline $v$ oscillation anomalies [1]. Each of these measurements lacks the significance to claim a discovery, but together they point toward the possible existence of non standard heavier sterile neutrino state(s) driving oscillations at small distances with $\Delta m^{2} \approx 1 \mathrm{eV}^{2}$ and relatively small mixing angles.

In order to give a definitive answer to the "sterile neutrino puzzle", a Short Baseline Neutrino (SBN) program is being developed at Fermi National Accelerator Laboratory (FNAL) [2]. It foresees the deployment along the Booster Neutrino Beam (BNB) of three detectors, all based on the Liquid Argon Time Projection Chamber (LAr-TPC) technique, namely: SBND (112 t active volume), MicroBooNE (89 t) and ICARUS T600 (476 t), at a distance of 110, 470 and $600 \mathrm{~m}$ respectively. Such configuration will allow simultaneous observations of neutrino interactions at different distances, by independently measuring both $v_{e}$ appearance and $v_{\mu}$ disappearance oscillation channels. In case of absence of oscillation, it is expected to have perfectly superimposed neutrino spectra at the different detectors; on the contrary any difference may imply a new physics signal. Sensitivity studies and MC simulations demonstrate the possibility to cover the anomaly parameter region with a $5 \sigma$ confidence level in three years of data taking $\left(\approx 6.6 \times 10^{20}\right.$ pot $)$ by all three LAr-TPC detectors.

\section{ICARUS T600 and the Liquid Argon TPC detection technique}

LAr-TPC detectors are well suited for experimental studies of neutrino physics, combining a massive and homogeneous target with excellent tracking and calorimetric capabilities. Originally proposed by C. Rubbia in 1977 [CERN-EP/77-08], this technology has been taken to full maturity with ICARUS T600, which still represents the largest LAr-TPC detector ever built.

The T600 detector is made of two identical cryostats each housing two faced TPCs sharing a common cathode [3]. They are filled with a total of $760 \mathrm{t}$ of ultrapure liquid argon corresponding to $470 \mathrm{t}$ of active mass. Each TPC has a maximal drift length of $1.5 \mathrm{~m}$, over which a constant electric field $E=500 \mathrm{~V} / \mathrm{cm}$ is present.

Charged particles interacting in the TPC produce both scintillation light and ionization electrons: the latter are drifted by the field to the anode, which is made by three parallel wire planes with different orientation $\left(0^{\circ} ; \pm 60^{\circ}\right), 3 \mathrm{~mm}$ apart and with a $3 \mathrm{~mm}$ pitch. Four planes of 8 " photomultipliers (PMTs), positioned behind the wire planes, detect the LAr scintillation light yielding information about the track arrival time. To this purpose VUV photons $(\lambda=128 \mathrm{~nm})$ are converted to visible light by means of a wavelengh shifter (TPB) deposited on the PMT windows.

The measured drift time, combined with the electron drift velocity $(v \approx 1.6 \mathrm{~mm} / \mu \mathrm{s}$ at $E=$ $500 \mathrm{~V} / \mathrm{cm}$ ) provides the event coordinate in the drift direction; the composition of the three views from the TPC wires yields the track projection on the anode plane. This information allows obtaining a full 3D reconstruction of the tracks, with a spatial resolution of $\approx 1 \mathrm{~mm}^{3}$.

ICARUS T600 took data from 2010 to 2013 in the Gran Sasso Laboratory (Italy), both with atmospheric neutrinos and with CNGS beam from CERN. The search for possible long-baseline 
sterile neutrino oscillations, resulted in no evidence of $v_{e}$ excess above the expected beam contaminations [4].

During the LNGS run two main important LAr-TPC features were demonstrated: the capability to attain unprecedented levels of liquid argon purity and the achievement of high efficient $e / \gamma$ separation. The LAr purification system, based on commercial Hydrosorb/Oxysorb ${ }^{\mathrm{TM}}$ filters, allowed obtaining, during the LNGS run, an average electron life-time better than $7 \mathrm{~ms}$ and a maximum value of $16 \mathrm{~ms}$ which translates into an impurity concentration lower than $20 \mathrm{ppt} \mathrm{O}_{2}$ equivalent [5]. Such a result represents a fundamental step towards the construction of a new generation of detectors, with drift paths many meters long.

The precise spatial and calorimetric reconstruction achievable in the T600, along with the possibility to measure local energy depositions $(d E / d x)$ wire by wire, allows the recognition of single and double minimum ionising particles events. This unique detection property of the LAr-TPC, which consents to identify unambiguosly individual $v_{e}$ events with high efficiency, was demonstrated by ICARUS T600 at LNGS with both CNGS events and atmospheric neutrinos. These latter cover the energy range of BNB neutrinos and represent a relevant test-bench to qualify neutrinos identification algorithm under development in view of operations at FNAL.

\section{ICARUS T600 overhauling at CERN}

The T600 detector is presently undergoing a significant overhauling process. This introduces new technological developments while at the same time maintaining the already achieved performance. This process is taking place at CERN in the framework of the Neutrino Platform activities (WA104). The overhauling covers important technical sections of the T600: the upgraded light detection system and the renovation of the read-out electronics represent the most important tasks.

In this new experimental phase, the T600 detector will operate at surface and will be subject to the interference of a huge cosmic background. Thus it is fundamental to recognize the signals related to the neutrino beam induced events. This requires a light detection system with very good features. New Hamamatsu photomultipliers R5912- MOD replace the outdated ETL 9357FLA units previously installed in the detector. A total of 400 devices have been tested and 360 of them are being mounted behind the 4 wire planes (90 each).

The new read-out electronics is based on the adoption of serial 2-bit ADCs, one per channel, in place of the multiplexed ones used in T600 at LNGS. The result consists in a faster read-out system and in a very compact set-up allowing for hosting both analog and digital electronics directly on the feed-through flanges. The main advantage is the synchronous sampling (400 ns) of all channels of the whole detector which is relevent for the $\mu$ momentum measurement by multiple scattering.

\section{SBN present status}

The MicroBooNE detector is already located $470 \mathrm{~m}$ from the BNB primary target. Its total mass is $170 \mathrm{t}$ while active mass is $89 \mathrm{t}$ of liquid argon. It consists of a single TPC with $2.5 \mathrm{~m}$ drift. Each anode consists of 3 wires planes. The scintillation light is decected by means of 32 photomultipliers ( 8 " diameter). The cryostat was successfully filled in mid-2015 and the detec- 

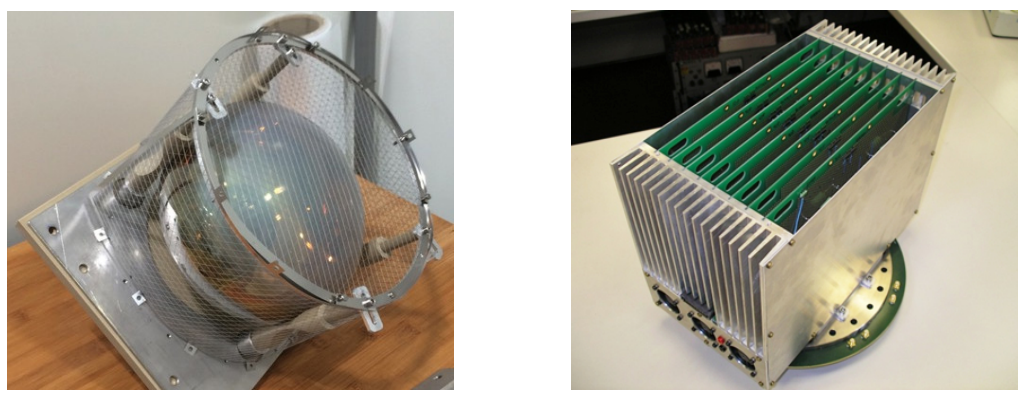

Figure 1: The upgraded light collection system with new PMTs (left) and the renovation of the read-out electronics with a compact lay-out (right) represent the most important tasks of the T600 overhauling.

tor is currently running and taking data addressing the MiniBooNE anomaly; it will continue as intermediate detector for the SBN program

The Short-Baseline Near Detector (SBND) is a $112 \mathrm{t}$ active volume LAr-TPC to be located only $110 \mathrm{~m}$ from the BNB neutrino source acting as near detector. It consists of 4 TPC with $2 \mathrm{~m}$ drift. Each anode consists of 3 wires planes. The scintillation light is decected by means of 120 photomultipliers ( 8 " diameter). The TPC assembly will begin in 2017 and the detector is expected to become operational in 2018.

The civil construction of the far site and building is rapidly progressing at FNAL. The overhauling of ICARUS T600 is almost complete ad the trasport is foreseen in early 2017. The installation and commissioning will follow, to start as soon as possible the data taking.

\section{Conclusions}

Fifty years after their introduction by B. Pontecorvo, sterile neutrios are still an open question in particle physics. The SBN program, in preparation at FNAL, is expected to definitely prove or exclude their existence and to find a solution to the observed short-baseline anomalies. The upgraded ICARUS T600 detector, together with MicroBooNE and SBND, will collect millions of events operating at $L / E \approx 1 \mathrm{~km} / \mathrm{MeV}$ at the BNB. The present anomaly parameter region will be covered with a $5 \sigma$ confidence level in three years of data taking.

\section{References}

[1] C. Giunti Sterile neutrino mixing now and in the next 10+ years, this conference.

[2] FNAL Proposal P-1052 (2014); arXiv:1312.7252 (2013); arXiv:1503.01520 (2015).

[3] S. Amerio et al. Design, construction and tests of the ICARUS T600 detector, Nucl. Instrum. Meth. A 527 (2004) 329-410.

[4] M. Antonello, et al. Experimental search for the "LSND anomaly" with the ICARUS detector in the CNGS neutrino beam, Eur. Phys. J. C (2013) 73:2345.

[5] M. Antonello, et al. Experimental observation of an extremely high electron lifetime with the ICARUS-T600 LAr-TPC, 2014 JINST 9 P12006. 